\title{
Estrategias tecnológicas en grupos con economías mixtas: una aproximación desde el conjunto lítico de Boyo Paso 2 (Pocho, Sierras de Córdoba, Argentina)
}

\section{Imanol Balena*}

\author{
Fecha de defensa: 29 de mayo de 2020 \\ Director: Dr. Matías E. Medina \\ Codirector: Dr. Gabriel E. López \\ Jurados: Dres. Juan P. Carbonelli y Federico Restifo
}

En esta tesis se presentan los resultados del análisis del conjunto lítico recuperado en las excavaciones del sitio arqueológico Boyo Paso 2, un asentamiento a cielo abierto localizado en el sector oriental del valle de Salsacate (Figura 1) que fue ocupado en repetidas ocasiones durante el período Prehispánico tardío (ca. 1500-360 años AP) por grupos que combinaban cultivos, caza-recolección y uso flexible del espacio (Medina, Pastor y Recalde, 2016). El propósito del estudio fue ampliar el conocimiento sobre las estrategias tecnológicas adoptadas en contextos donde la incorporación de cultivos no restringió la movilidad estacional del grupo ni el tiempo invertido en la caza-recolección, una temática escasamente contemplada en la arqueología argentina por su baja visibilidad arqueológica y ausencia de marcos interpretativos adecuados. En este sentido, el estudio tuvo como objetivo específico reconocer qué etapas de la elaboración de instrumentos estaban representadas en el sitio, observar la distribución de materias primas en el conjunto y determinar la forma en que éstas fueron aprovechadas, vinculando estos aspectos con parámetros sociales y económicos de los grupos en estudio. El énfasis, en consecuencia, se centró en establecer la relación entre las estrategias tecnológicas y el desarrollo de un sistema de subsistencia de amplio espectro, caracterizado por una movilidad residencial estacional y eventos de ocupación y reocupación del espacio.

\section{Área de estudio y sitio}

El conjunto montañoso conocido como Sierras de Córdoba comprende tres cordones serranos -el cordón central, el oriental y el occidental- que se desarrollan en forma paralela por más de 600 km, entre los $29^{\circ}$ y los $33^{\circ} 44^{\prime}$ de latitud Sur (Medina, 2008). Los valles occidentales, emplazados entre el cordón serrano central y el occidental, reciben la denominación genérica de "Traslasierra" aunque pueden reconocerse dos segmentos separados por la pampa de Pocho. Al norte se halla el extenso valle de Salsacate, donde está localizado el sitio Boyo Paso 2.

* División Arqueología, Facultad de Ciencias Naturales y Museo, Universidad Nacional de La Plata (UNLP) - CONICET. Av. 122 y 60, Laboratorio 102 (CP B1900FWA), La Plata, Buenos Aires, Argentina. Email: imanol.balena@gmail.com
En estos paisajes serranos, en las diferentes zonas montañosas ubicadas entre 600 y 1.000 o 1.400 m.s.n.m. la cubierta vegetal de los valles, así como los piedemontes, laderas bajas y quebradas tributarias, corresponde al Bosque Serrano o Chaco Serrano (Demaio, Karlin y Medina, 2002; Luti et al., 1979). La fauna muestra las características comunes a diferentes ambientes chaqueños con vegetación boscosa, con algunas especies que pudieron resultar de importancia económica para las poblaciones prehistóricas. Sobre el cordón central se disponen espacios dilatados que reciben la denominación local de "pampas" o "pampillas" que pueden alcanzar alturas por encima de los $2.000 \mathrm{~m}$. Estas pampas, zoogeográficamente se corresponden con el Distrito Subandino (Ringuelet, 1961), destacándose los elementos faunísticos de origen andinopatagónico y con características ambientales comunes que permiten diferenciarlas de los entornos deprimidos.

La evidencia regional y paleoecológica actualmente disponible ha permitido plantear que los sitios a cielo abierto asignados al período Prehispánico tardío no fueron de uso continuo sedentario, sino que correspondían a concentraciones estivales de unidades domésticas para realizar actividades agrícolas y de recolección junto con otras tareas que requerían de cierta estabilidad residencial, como la manufactura de artefactos cerámicos. Finalizadas estas tareas, el grupo co-residencial se dispersaba con el fin de aprovechar al máximo frutos silvestres y productos de caza disponibles en distintos puntos del espacio serrano (Medina et al., 2016). Las investigaciones en el sitio Boyo Paso 2 fueron importantes dentro de esta nueva perspectiva, por cuanto permitieron definir las características de la arquitectura doméstica y la dinámica de ocupación de los asentamientos residenciales tardíos. En tal sentido, las intervenciones de área abierta revelaron dos pisos de ocupación con numerosos orificios de postes, macro-restos de frutos comestibles y artefactos todavía útiles o con alta recurrencia de remontaje (Medina, Balena y Rivero, 2019). La diversidad taxonómica y el tamaño de los conjuntos faunísticos, junto a la alta densidad de restos cerámicos y líticos recuperados en estratigrafía, fueron indicativos de estadías de varios meses en el sitio, con una alta incidencia de actividades cinegéticas. Por su parte distintos indicadores faunísticos de estacionalidad, junto con evidencias de actividades agrícolas y de recolección, sugieren que la ocupación del asentamiento al menos coincidió con la primavera-verano, momento del año en que debía realizarse la siembra, cosecha y/o que los recursos silvestres estaban disponibles en los alrededores del sitio (Medina et al., 2016). Por otro lado, la existencia de estructuras habitacionales se infirió a partir de la presencia de numerosos agujeros de postes, que al casi superponerse, desarrollar diámetros variables y no tener 


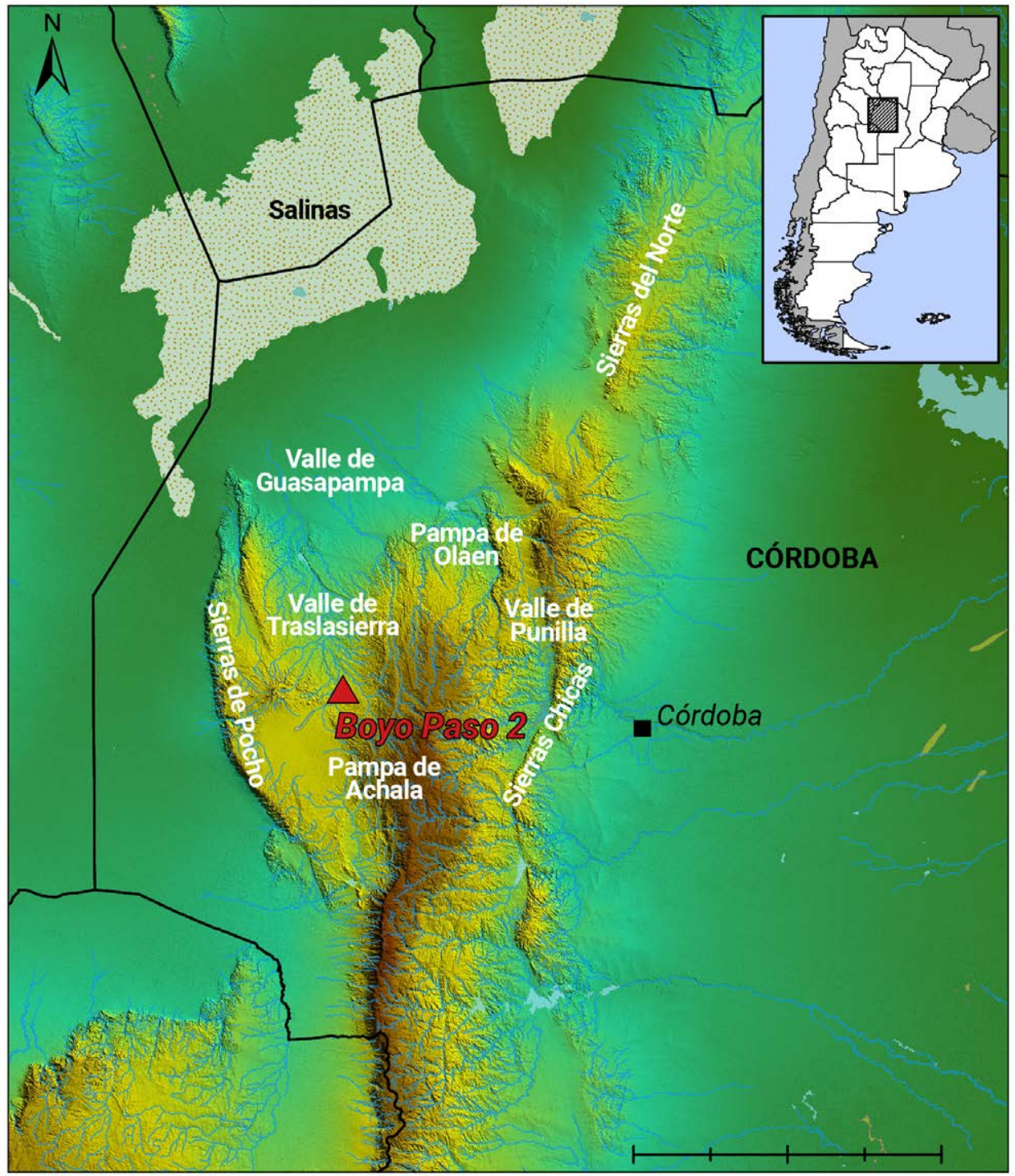

Figura 1. Ubicación del área de estudio y del sitio Boyo Paso 2.

un patrón claro en cuanto a su disposición, pueden representar distintos eventos ocupacionales. El rango de variación de los fechados obtenidos ca. 1500 - 700 años AP, sumado a conjuntos polínicos dominados por plantas herbáceas comúnmente descriptas como indicadoras de un continuo disturbio antrópico, refuerza este planteo (Medina et al., 2019). En consecuencia, Boyo Paso 2 fue interpretado como un palimpsesto formado por sucesivas ocupaciones estivales de unas pocas familias para realizar actividades diversas propias de los espacios residenciales, en el marco de una estrategia económica mixta que combinaba cultivos y caza-recolección junto con un uso estacional y diversificado del paisaje.

\section{Metodología y marco teórico}

La muestra analizada del sitio Boyo Paso 2 correspondió a todos los artefactos tallados, núcleos y desechos de talla recuperados, los cuales contabilizaron un total de 2.305 piezas (Tabla 1). Los instrumentos de molienda hallados, formatizados por abrasión y pulido como las manos de molienda, conanas y hachas, no se tuvieron en cuenta. Los materiales recuperados fueron considerados como parte de un mismo "componente" formado por la sucesión de ocupaciones dentro del rango cronológico del período Prehispánico tardío y analizados en conjunto, sin distinguir por pisos de ocupación.

Durante el análisis se describió el tipo de materias primas y las características tecno-morfológicas de cada pieza lítica, siguiendo los lineamientos de Aschero $(1975,1983)$ y clasificando los artefactos en grupos tipológicos. La identificación de las rocas se realizó en base a estudios macroscópicos a simple vista y lupa binocular. En casos específicos se realizaron cortes petrográficos para confirmar las identificaciones. Una vez identificadas las rocas y/o minerales, las potenciales canteras de aprovisionamiento fueron rastreadas en las cartas geológicas y la literatura existente (Heider, Rivero y Baldo, 2015), clasificando las rocas como locales y/o no locales. 
El marco teórico a partir del cual se abordó la información obtenida fue el de la organización tecnológica (sensu Nelson, 1991). Este enfoque postula que la toma de decisiones con respecto a la tecnología lítica se ve reflejada en lo que se denominan estrategias tecnológicas, las cuales son vistas como procesos de resolución de problemas que responden a las condiciones creadas en el inter-juego de la sociedad con su ambiente (Nelson, 1991). En particular, este trabajo hizo foco en la movilidad del grupo en cuestión, la disponibilidad y distribución de las materias primas y en la naturaleza de los recursos a explotar como factores que pudieron incidir en la toma de decisiones.

\begin{tabular}{|c|c|c|}
\hline Clase técnica & N & $\%$ \\
\hline Núcleos & 40 & 1,74 \\
Desechos de talla & 2142 & 93,01 \\
Artefactos formatizados & 67 & 2,91 \\
FNRC & 11 & 0,47 \\
Puntas de proyectil & 45 & 1,95 \\
\hline Total & $\mathbf{2 3 0 5}$ & $\mathbf{1 0 0}$ \\
\hline
\end{tabular}

Tabla 1. Características generales del conjunto lítico de Boyo Paso 2. Referencia: FNCRC (filos naturales con rastros complementarios).

\section{Resultados y conclusiones}

Los grupos humanos que habitaron el valle de Salsacate durante el período Prehispánico tardío y, en particular, Boyo Paso 2, se valieron de una combinación de estrategias tecnológicas tendientes tanto a la expeditividad como a la conservación para obtener recursos del ambiente. Las características del conjunto lítico del sitio mostraron cierta correlación con otros asentamientos de la región ubicados en el mismo rango cronológico e integrados dentro de los circuitos de movilidad de las sociedades tardías (Balena, Heider y Medina, 2018; Pastor, 2007; Rivero, 2009). Sin embargo, Boyo Paso 2 presentó características distintivas que lo diferenciaron de los demás. Por ejemplo, se observó una mayor representación de rocas locales como la andesita, un material ausente o con muy baja representatividad en los conjuntos líticos de otros asentamientos, aun de aquellos cercanos al sitio aquí abordado (Pastor, 2007). También se determinó una menor proporción del cuarzo, el cual es dominante en los restantes sitios de la región (Balena et al., 2018; Rivero, 2009). De esta manera, resulta coherente que muchas características evidenciadas en los conjuntos líticos del Prehispánico tardío no coincidan exactamente con los registrados en Boyo Paso 2, ya que es un efecto lógico de las particularidades que inevitablemente tendrá cada conjunto y que son producto de la interacción diaria entre la toma de decisiones en la organización tecnológica y el contexto ecológico y social más general en el que se encuentran insertas (Gaál, 2014).
De esta manera, a partir de la evidencia relevada en Boyo Paso 2 fue posible señalar que el proceso de manufactura de artefactos líticos se caracterizó por una baja inversión de energía, indicando que estos grupos habrían utilizado una estrategia expeditiva aprovechando las ventajas de las fuentes cercanas, como el cuarzo y la andesita. Otro factor que influyó fue la predictibilidad de cierto tipo de recursos explotados, en particular aquellos que permanecieron inmóviles y con ubicación conocida, ya que los instrumentos de procesamiento son los que evidenciaron la implementación de una estrategia tendiente a la expeditividad. En este sentido, la idea de una baja inversión de energía se sustentó en base a las características de los instrumentos, núcleos y desechos de talla. Los primeros no reflejaron un uso intensivo y tampoco se registró el uso de formas bases estandarizadas, observándose una baja energía en su manufactura y la presencia de filos aun activos al momento de su descarte o depositación. Esta situación, sumada a la presencia de núcleos con posibilidad de seguir siendo explotados, o lascas con tamaños apropiados para ser utilizadas como formas bases, refuerza la idea del desarrollo de estrategias tecnológicas expeditivas y de un uso que no buscó un máximo aprovechamiento de las materias primas (Hocsman 2006; Parry y Kelly, 1987), aunque también puede sugerir la conformación de un espacio con presencia de materia prima en forma de artefactos para posteriores visitas (Binford, 1979). Por su parte, las estrategias conservadas se aplicaron en los materiales de mejor calidad para la talla, los cuales se utilizaron en la confección, casi exclusivamente, de puntas de proyectil. Estos instrumentos, evidenciaron una buena inversión de energía en su mantenimiento y elaboración, por lo que se deduce que los pobladores de Boyo Paso 2 buscaban generar instrumentos confiables y que no fallaran al momento de ser utilizados.

De esta forma, el análisis del conjunto artefactual lítico de Boyo Paso 2 permitió un mayor entendimiento de las estrategias tecnológicas prehispánicas implementadas por grupos que desarrollaron un nicho económico de amplio espectro, acompañado de un grado variable de movilidad residencial, momentos de dispersión / agregación de los grupos co-residentes y procesos estacionales de abandono de las prácticas productivas para abastecerse de recursos de caza y recolección (Medina et al., 2016). Se espera que este corpus de datos sea útil para interpretar la organización tecnológica en casos arqueológicos del Holoceno tardío del centro y otras áreas del país, donde el abandono contingente de las estrategias productivas pudo haber sido más frecuente que lo tradicionalmente esperado, sobre todo cuando no existe aún entre los arqueólogos un consenso claro respecto a qué factores pudieron estructurar los conjuntos artefactuales líticos y el patrón de asentamiento antes de la adopción generalizada de plantas cultivadas (Kelly, 1992; Odell, 1998, entre otros).

\section{Referencias Citadas}

» Aschero, C.A. (1975). Ensayo para una clasificación morfológica de artefactos líticos aplicada a estudios 
tipológicos interpretativos. Informe inédito presentado al CONICET. Manuscrito.

» Aschero, C.A. (1983). Ensayo para una clasificación morfológica de artefactos líticos. Apéndices A y $\mathrm{B}$. Apunte inédito para la cátedra de Ergología y Tecnología. Facultad de Filosofía y Letras. Universidad de Buenos Aires. Manuscrito.

» Balena, I., Heider, G. y Medina, M. (2018). Tecnología lítica entre las sociedades del Período Prehispánico Tardío (Sierras de Córdoba, Argentina). Mundo de Antes, 12(1), 81-105.

» Binford, L. R. (1979). Organization and formation processes: looking at curated technologies. Journal of Anthropological Research, 35(3), 255-273.

» Demaio, P.; Karlin, O. y Medina, M. (2002). Árboles nativos del centro de la Argentina. Buenos Aires: Literature of Latin American (LOLA).

" Gaál, E. (2014). Decisiones tecnológicas y producción lítica en el sur del valle de Yocavil (pcia. de Catamarca). Un estudio comparativo de conjuntos artefactuales tempranos y tardíos. (Tesis de Licenciatura inédita). Universidad de Buenos Aires, Argentina.

" Heider G., Rivero, D. y Baldo, E. (2015). Rocas de uso arqueológico en Sierras Centrales. Fuentes de recursos líticos identificadas y potenciales en las provincias de Córdoba y San Luis, Argentina. Revista de Antropología del Museo de Entre Ríos, 1(2), 55-72.

» Hocsman, S. (2006). Producción lítica, variabilidad y cambio en Antofagasta de la Sierra. (Tesis de Doctorado inédita). Universidad Nacional de La Plata, Argentina.

» Kelly, R. L. (1992). Mobility/sedentism: concepts, archaeological measures, and effects. Annual Review of Anthropology, 21(1), 43-66.

" Luti, R., Bertran de Solís, M., Galera, F., Muller de Ferreira, N., Berzal, M., Nores, M., Herrera, M. y Barrera, J. (1979). Vegetación. En Vazquez, J., R. Miatelo y M. Roque (Eds.), Geografía Física de la Provincia de Córdoba (pp. 297-368).
Buenos Aires: Editorial Boldt.

" Medina, M. E. (2008) Diversificación Económica y Uso del Espacio en el Tardío Prehispánico del Norte del Valle de Punilla, Pampa de Olaen y Llanura Noroccidental (Córdoba, Argentina). (Tesis Doctoral inédita). Universidad de Buenos Aires, Argentina.

» Medina, M., Balena, I. y Rivero, D. (2019). Proyectiles y procesos de intensificación: una aproximación desde Boyo Paso 2, ca. 1500-75 AP (Sierras de Córdoba, Argentina). Chungara, Revista de Antropología Chilena, 51(4), 517-529.

» Medina, M., Pastor, S. y Recalde, M. A. (2016). The archaeological landscape of late prehispanic mixed foraging and cultivation economy (Sierras of Cordoba, Argentina). Journal of Anthropological Archaeology, 42, 88104.

"Nelson, M. C. (1991). The study of technological organization. Archaeological Method and Theory, 3, 57-100.

» Odell, G. H. (1998). Investigating Correlates of Sedentism and Domestication in Prehistoric North America. American Antiquity, 63(4), 553-571.

» Parry, W. J. y Kelly, R. L. (1987). Expedient core technology and sedentism. En J. K. Johnson y C. A. Morrow (Eds.), The Organization of Core Technology (pp. 285-312). Boulder and London: Westview Press.

"Pastor,S. (2007). Antecedentes de la adopción de la agricultura entre las sociedades prehispánicas de las Sierras Centrales de Argentina (ca. 2500-1000 AP). En Libro de Resúmenes extendidos del II Encuentro de Discusión Arqueológica del Nordeste Argentino (pp. 93-98). Santa Fe: Dirección de Cultura de la Municipalidad de Santo Tomé, Ministerio de Innovación y Cultura de la Provincia de Santa Fe.

" Ringuelet, R. (1961). Rasgos fundamentales de la zoogeografía de la Argentina. Physis, 63,151-170.

» Rivero, D. (2009). Ecología de Cazadores-Recolectores del sector central de las Sierras de Córdoba (Rep. Argentina). Oxford: Archaeopress. 